\title{
Rate-one Space Frequency Block Codes with Maximum Diversity Gain for MIMO-OFDM
}

\author{
Lei Shao \\ Dept. of Electrical Engineering \\ University of Washington \\ Seattle, WA 98195-2500 \\ Email: 1s7@ee.washington.edu
}

\author{
Sumit Roy \\ Wireless Technology Development \\ Intel Labs \\ Hillsboro, OR,97124 \\ Email: sumit.roy@intel.com
}

\author{
Sumeet Sandhu \\ Intel Corporation \\ M/S RNB-6-49 \\ 2200 Mission College Blvd \\ Santa Clara, CA 95052 \\ Email: sumeet.sandhu@intel.com
}

\begin{abstract}
We proposed a novel rate-1, space-frequency (SF) code for an Orthogonal Frequencey Division Multiplexing (OFDM) system with transmit $(M)$ and receive $(N)$ antennas that achieves the maximum diversity attainable over frequencyselective channels. Since the SF code symbol is transmitted in one OFDM block duration, it has smaller processing delay than previously proposed Space-Time-Frequency (STF) block codes.
\end{abstract}

\section{INTRODUCTION}

The demand for high speed data services to residential and enterprise locations has led to 'last mile' fixed broadband wireless access (BWA) in $2-11 \mathrm{GHz}$ band. This is particularly apropos for regions with current low penetration of cable/telephony loop plant (such as in developing nations) and allows bypassing the costly investment in such wired infrastructures. The IEEE 802.16a Task Group for Wireless Metropolitan Area Networks (MAN) has recently standardized the air interface for wireless access to a stationary receiver from a base station(BS) [1], [2].

In fixed BWA, deep fades occur that can persist over a significant period of time. Further, such wide-area wireless channels encounter significant dispersion due to multipath propagation that limits the maximum achievable rates. Since fixed BWA competes with cable modems and asynchronous DSL where the channel is static and non-fading, such system designs must counteract these key challenges and provide high-data-rate access at almost wireline quality. Hence, multiple antennas at both transmit and receive ends of a wireless link resulting in a multiple-input multiple output (MIMO) channel has been recognized as an important system component towards meeting the desired data rate requirements. Point-to-point MIMO schemes ideally allow both high-rate transmission and exploiting of the path diversity available due to multipath present between any transmit-receive pair in a MIMO set-up; in practice there exists a fundamental tradeoff between spatial multiplexing that increases the achievable rate and diversity gain that enhances reliability [8]. In a multiplexing approach such as the BLAST scheme [7],the message bits to transmitted signal mappings minimize the redundancy between the various antenna signals to achieve maximum data rate. In contrast, the space-time coding (STC) approach of [14] introduces sufficient redundancy in an effort to maximize the diversity gain (and minimize bit error rate (BER)).

Orthogonal Frequencey Division Multiplexing (OFDM) is a multi-carrier transmission scheme that is well-recognized for it's potential for attaining high rate transmission over frequency selective channels. OFDM eliminates the effects of inter-symbol interference due to multipath by inserting sufficient cyclic prefix (CP); this also enables simple tone-by-tone-basis equalization at the receiver. Thus a combination of MIMO and OFDM (MIMO-OFDM) appears particularly promising for high-rate, fixed BWA. Recently, there has been great interest in MIMO-OFDM[3][4][5][9][11] [13][18][17]. For frequency-selective fading channels, STBCOFDM modulation[12] have been (naturally) proposed to preserve the orthogonality of STBCs (that leads to a simple receiver) while enabling high data rates. However, the channels need to be quasi-static over several OFDM symbol blocks.Moreover, since the ST encoding is applied at individual subcarriers, only maximum spatial diversity gain but not frequency diversity is achieved. In order to exploit the frequency selectivity provided by the channel, coding across the subcarriers is needed. [9] seems to be one of earliest papers on space-frequency trellis coding. Later, [11] suggested the idea of space-frequency encoded OFDM that uses Alamouti's code in both the space and frequency domains. This SFBCOFDM achieves a diversity gain of $2 N$ in the 2 transmit, $N$ receive antenna case, independent of the channel order; i.e. it does not exploit the frequency selectivity inherent in the channel.

Among the contributions to 802.16a, the STFBC-OFDM code proposed by [13] appears promising. At the transmitter, an OFDM block is cyclically shifted on a subcarrier basis to produce another OFDM block ${ }^{1}$. Both are transmitted in two successive OFDM block intervals using the Alamouti code mapping. This code can simultaneously obtain spacetime diversity and order- 2 frequency diversity gain; however, the frequency-diversity gain does not increase with the channel order in this technique. Moreover, even with 2 transmit antenna case, the code rate is 0.5 and is possibly lower for larger

\footnotetext{
${ }^{1}$ The amount of cyclic shift is based on the stochastic properties of the channel environment.
} 
number of transmit antennas; thus it trades achievable rate for limited (2nd order) diversity gain. Further, choice of the cyclic shift of the replica symbol implies the need for a feedback path since this is determined on a subcarrier basis by the receiver.

In [3], the design criteria for space-frequency coding was established. It was shown that the figure of merit for designing good space-frequency codes is vastly different from those for space-time codes in narrowband fading channels. For example, employing known space-time codes as space-frequency codes by coding across space and frequency (rather than across space and time) in general provides spatial diversity but fails to exploit the available frequency diversity [3]. The case of two transmit and one receive antenna for a two-tap channel was considered in [4]. Although a full diversity code was designed, the design criteria imposed limits on the achievable code rate leading to a low rate $1 / 4$ space-frequency code.

In [5], a novel space-time-frequency (STF) block code for multiple antenna OFDM transmission over frequency-selective Rayleigh fading channels was proposed. Incorporating subcarrier grouping and choosing appropriate system parameters, the authors first convert their system into a set of grouped STF systems. This enables simplified STF block coding within each group. A design criteria for grouped STF (GSTF) block coding was proposed and existing ST coding techniques were exploited to construct STF block codes. The resulting codes were shown capable of achieving both maximum diversity and coding gain, while affording low-complexity decoding. However, since the authors use orthogonal space-time block code as the component S-T code, for number of transmit antennas greater than two, the resulting code rate of STFBC will fall below $3 / 4$, leading to spectral inefficiency. Another disadvantage of [5] is that it requires the channel to be constant during $2 M(M \geq 4)$ OFDM symbol times for $M$ transmit antennas implying longer processing delay. Recently, another kind of space-time-multipath coding method which uses digital phase sweeping (DPS) has been proposed in [18]. This overcomes the drawback of [5] of rate loss and can guarantee maximum diversity gain and achieve good coding gain. When combined with OFDM, DPS converts the $M$ transmit-antenna system where each frequency-selective channel has L taps, to a single transmit-antenna system, where the equivalent channel has $M \times L$ taps.

In this paper, we present a new coding scheme that maximizes diversity gain in MIMO-OFDM systems over frequency-selective channels; like [18], our space-frequency block code (SFBC) also guarantees the maximum possible space-multipath diversity without rate loss for any number of transmit antennas and requires that the channel be constant only for one OFDM symbol time. It is of interest to note that although both [18] and our SFBC-OFDM are designed independently and the two code matrices have different structures, the complexity and performance of these two schemes are very close to each other. Table I summarizes the above and provides a comparison among various ST/SF/STF block coded OFDM schemes.
TABLE I

COMPARISON AMONG VARIOUS ST/SF/STF BLOCK CODED OFDM SCHEMES

\begin{tabular}{|c|c|c|c|}
\hline methods & $\begin{array}{l}\text { diversity gain } \\
\text { (DG) }\end{array}$ & $\begin{array}{l}\text { code rate } \\
\text { (CR) }\end{array}$ & comments \\
\hline $\begin{array}{c}\text { STBC-OFDM } \\
(\mathrm{M}=2)\end{array}$ & $2 \mathrm{~N}$ & 1 & $\begin{array}{c}\text { if } M>2, \\
D G=M N \\
C R<3 / 4\end{array}$ \\
\hline $\begin{array}{l}\text { SFBC-OFDM[11] } \\
\qquad(\mathrm{M}=2)\end{array}$ & $2 \mathrm{~N}$ & 1 & $\begin{array}{c}\text { if } M>2, \\
D G=M N \\
C R<3 / 4\end{array}$ \\
\hline $\begin{array}{l}\text { STFBC-OFDM[13] } \\
\qquad(\mathrm{M}=2)\end{array}$ & $4 \mathrm{~N}$ & 0.5 & $\begin{array}{c}\text { if } M>2, \\
D G=2 M N\end{array}$ \\
\hline $\begin{array}{c}\text { STFBC-OFDM[5] } \\
(\mathrm{M}=2)\end{array}$ & $2 \mathrm{NL}$ & 1 & $\begin{array}{c}\text { if } M>2, \\
D G=M N L \\
C R<3 / 4\end{array}$ \\
\hline DPS $[18]$ & MNL & 1 & $\forall M, N$ \\
\hline SFBC-OFDM proposed & MNL & 1 & $\forall M, N$ \\
\hline
\end{tabular}

\section{Design CRiteria And Code Construction}

We adopt the design criteria proposed in [4] for a MIMOOFDM system with $M$ transmit and $N$ receive antennas and $N_{c}$ sub-carriers, where $N_{c}>>M, N$. Let $C$ and $E$ be two different space-frequency code words represented by matrices of size $M \times N_{c}$. Assuming that the MIMO channel consists of $L$ (matrix) taps, an upper bound on the expected pairwise error probability (averaged over the general Rayleigh fading channel realizations) was derived. For the special case of no spatial fading correlation and a uniform power delay profile, the upper bound can be simplified as

$$
P(C \rightarrow E) \leq \prod_{i=0}^{\operatorname{rank}(S)-1}\left(1+\lambda_{i}(S) \frac{\rho}{4}\right)^{-N}
$$

where $\rho$ is the average signal-to-noise ratio,$\lambda_{i}(S)$ is the $i$-th nonzero eigenvalue of $S . S=G(C, E) G^{H}(C, E)$ has dimension $N_{c} \times N_{c}$ where $G(C, E)$ is the $N_{c} \times M L$ matrix $G(C, E)=\left[\begin{array}{llll}(C-E)^{T} & D(C-E)^{T} & \cdots & D^{L-1}(C-E)^{T}\end{array}\right]$ and $D=\operatorname{diag}\left\{e^{-j \frac{2 \pi}{N_{c}} k}\right\}_{k=0}^{N_{c}-1}$. In this paper, we restrict our attention to this special case. For $N_{c}>M L$, in order to achieve $M N L$-fold diversity, appropriate code design is needed to ensure that not only the $M \times N_{c}$ error matrix $(C-E)$ is of full rank over all distinct $\{C, E\}$ pairs, but the stacked matrix $G(C, E)$ always has full rank as well.

\section{A. Code Construction}

For rate-one space-frequency block codes, the number of information symbols mapped into the space-frequency code matrix is equal to the number of subcarriers $N_{c}$. We choose $N_{c}=M \times L \times G$, where $G$ is the number of groups the subcarriers are divided into (see Fig. 1 and Fig. 2 ).

First, the $N_{c} \times 1$ vector of input symbols $\mathbf{s}=$ $\left[\begin{array}{llll}\mathbf{s}_{0}^{T} & \mathbf{s}_{1}^{T} & \cdots & \mathbf{s}_{G-1}^{T}\end{array}\right]^{T}$ is divided into $G$ groups of size $M L \times 1$ vectors $\left\{\mathbf{s}_{g}\right\}_{g=0}^{G-1}$. Next, each $\mathbf{s}_{g}$ is left-multiplied by the same constellation-rotation (CR) precoder $\Theta$ [6] of dimension $M L \times M L$ to produce size $M L$-vector $\mathbf{v}_{g}=\Theta \mathbf{s}_{g}=$ 


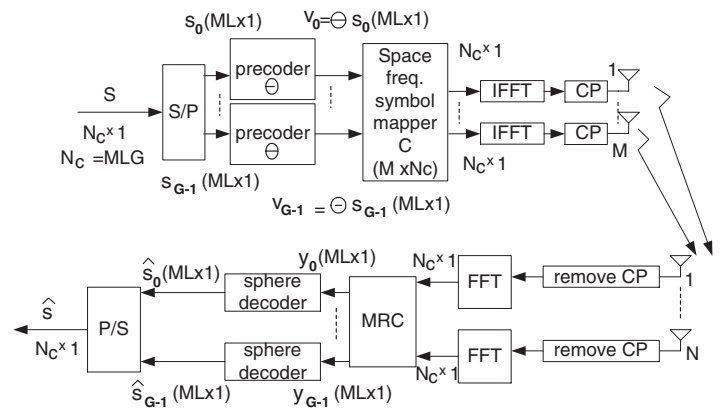

Fig. 1. Baseband multiple antenna OFDM system model

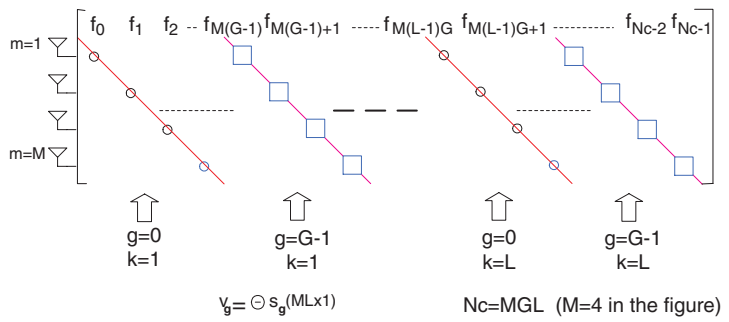

Fig. 2. Structure of Rate-one space-frequency block code matrix C

$\left[\theta_{1}^{T} \mathbf{s}_{g}, \cdots \theta_{M L}^{T} \mathbf{s}_{g}\right]^{T}$, where $\theta_{i}^{T}$ denotes the $i$ th row of $\Theta$. Then we divide each $\mathbf{v}_{g}$ into $L M \times 1$ subvectors; each subvector is then used to create a $M \times M$ diagonal matrix $D_{\mathbf{s}_{g}, k}=$ $\operatorname{diag}\left\{\theta_{M \times(k-1)+1}^{T} \mathbf{s}_{g}, \cdots, \theta_{M \times k}^{T} \mathbf{s}_{g}\right\}$, for $k=1, \cdots L$. The $L$ submatrices are regarded to be in the same group.

Finally, we "interleave" the submatrices from the $G$ groups (a total of $G \times L$ diagonal matrices) to get the $M \times N_{c}$ spacefrequency matrix

$C=\left[D_{\mathbf{s}_{0}, 1}, \cdots, D_{\mathbf{s}_{G-1}, 1}, \cdots, \cdots, D_{\mathbf{s}_{0}, L}, \cdots, D_{\mathbf{s}_{G-1}, L}\right]$

as shown in Fig.2, where $\left\{\mathbf{f}_{i}\right\}_{i=0}^{N_{c}-1}$ denote the $N_{c}$ subcarriers. As a result, submatrices corresponding to successive symbols in the same group are equi-spaced in the codeword $C$. The resulting design possesses the property that successive symbols from the same group transmitted from the same antenna are at a frequency "distance" that is multiples of $M G$ subcarrier spacings in order to exploit the diversity in frequency-selective channels. For known channel order and uniform power delay profile, the channel frequency responses on subcarriers spaced at multiple of $M G$ are uncorrelated as proved in [13].Therefore, the $L$ symbols from the same group transmitted from the same antenna experience uncorrelated fading.

In our SFBC, we make use of the following desirable property of $\Theta$ : for all distinct pairs $\left\{\mathbf{s}_{g}, \tilde{\mathbf{s}}_{g}\right\}$ and $\mathbf{v}_{g}=\Theta \mathbf{s}_{g}$, and $\tilde{\mathbf{v}}_{g}=\Theta \tilde{\mathbf{s}}_{g}$, the corresponding error vector $\mathbf{e}_{g}=\left(\mathbf{v}_{g}-\tilde{\mathbf{v}}_{g}\right)$ has all nonzero elements.

Thus if we generate $D_{\tilde{\mathbf{s}}_{g}, k}, k=1, \cdots L$ from $\tilde{\mathbf{s}}_{g}$, then the $L$ diagonal error matrices $\left(D_{\mathbf{s}_{g}, k}-D_{\tilde{\mathbf{s}}_{g}, k}\right)$ have all diagonal elements nonzero. Therefore, all distinct pairs $\left\{\mathbf{s}_{g}, \tilde{\mathbf{s}}_{g}\right\}$, give rise to $L$ full rank diagonal error matrices $\left(D_{\mathbf{s}_{g}, k}-D_{\tilde{\mathbf{s}}_{g}, k}\right)$; this property will be used later in our proof.
Lemma 1: The space-frequency codes proposed can achieve the maximum diversity gain of $M N L$.

Proof From (1), this is equivalent to proving that $\operatorname{rank}(S)=$ $M L$. Since $S=G(C, E) G^{H}(C, E)$ and $\operatorname{rank}(S)=$ $\operatorname{rank}(G(C, E))=\operatorname{rank}\left(G(C, E)^{T}\right)$, it suffices to show that $\operatorname{rank}\left(G(C, E)^{T}\right)=M L$, where $G(C, E)^{T}$ is a $M L \times N_{c}$ matrix

$$
G(C, E)^{T}=\left[\begin{array}{c}
(C-E) \\
(C-E) D \\
\vdots \\
(C-E) D^{L-1}
\end{array}\right]
$$

and $D=\operatorname{diag}\left\{e^{-j \frac{2 \pi}{N_{c}} k}\right\}_{k=0}^{N_{c}-1}$. For the proof, we will assume that the number of taps is known.

Consider the $N_{c} \times 1$ vectors $\mathbf{s}=\left[\begin{array}{llll}\mathbf{s}_{0}^{T} & \mathbf{s}_{1}^{T} & \cdots & \mathbf{s}_{G-1}^{T}\end{array}\right]^{T}$, and $\tilde{\mathbf{s}}=\left[\begin{array}{llll}\tilde{\mathbf{s}}_{0}^{T} & \tilde{\mathbf{s}}_{1}^{T} & \ldots & \tilde{\mathbf{s}}_{G-1}^{T}\end{array}\right]^{T}$ such that $\mathbf{s} \neq \tilde{\mathbf{s}}$; then there exists $\mathbf{s}_{g} \neq \tilde{\mathbf{s}}_{g}$ for some $g \in\{0, \cdots, G-1\}$. Without loss of generality, let $\mathbf{s}_{0} \neq \tilde{\mathbf{s}}_{0}$.

Define the diagonal $M \times M$ matrix $A_{(k-1) G+g+1}=D_{\mathbf{s}_{g}, k}-$ $D_{\tilde{\mathbf{s}}_{g}, k}, k=1, \cdots, L ; g=0, \cdots, G-1$.

Thus, $C-E=\left[\begin{array}{lll}A_{1} & \cdots & A_{G L}\end{array}\right]$. On the other hand, we can divide the diagonal matrix $D=\operatorname{diag}\left\{e^{-j \frac{2 \pi}{N_{c}} k}\right\}_{k=0}^{N_{c}-1}$ into $G L$ $M \times M$ diagonal sub-matrices $\left\{D_{i}\right\}_{i=1}^{G L}$.

Thus, we get

$$
\begin{aligned}
(C-E) D^{i} & =\left[\begin{array}{lll}
A_{1} & \cdots & A_{G L}
\end{array}\right]\left[\begin{array}{ccc}
D_{1}^{i} & 0 & \cdots \\
0 & \ddots & 0 \\
0 & \cdots & D_{G L}^{i}
\end{array}\right] \\
& =\left[\begin{array}{llll}
D_{1}^{i} & \cdots & D_{G L}^{i}
\end{array}\right]\left[\begin{array}{ccc}
A_{1} & 0 & \cdots \\
0 & \ddots & 0 \\
0 & \cdots & A_{G L}
\end{array}\right]
\end{aligned}
$$

To get the second equation, we used the fact that both $A_{j}$ and $D_{j}^{i}$ are diagonal matrices. Therefore,

$$
\begin{aligned}
G(C, E)^{T} & =\left[\begin{array}{cccc}
I_{M} & I_{M} & \cdots & I_{M} \\
D_{1} & D_{2} & \cdots & D_{G L} \\
\vdots & \vdots & \vdots & \vdots \\
D_{1}^{L-1} & D_{2}^{L-1} & \cdots & D_{G L}^{L-1}
\end{array}\right] \\
& \times\left[\begin{array}{ccc}
A_{1} & 0 & \cdots \\
0 & \ddots & 0 \\
0 & \cdots & A_{G L}
\end{array}\right]
\end{aligned}
$$

As previously shown that over all distinct pairs $\left\{\mathbf{s}_{0}, \tilde{\mathbf{s}}_{0}\right\}$, we have $\mathrm{L}$ full rank diagonal error matrices $A_{(k-1) G+1}=$ $\left(D_{\mathbf{s}_{0}, k}-D_{\tilde{\mathbf{s}}_{0}, k}\right)$, for $k=1, \cdots L$. Thus, in $G(C, E)^{T}$, we can find a $M L \times M L$ submatrix which is the product of two 
other $M L \times M L$ matrices as follows:

$$
\begin{gathered}
{\left[\begin{array}{cccc}
I_{M} & I_{M} & \cdots & I_{M} \\
D_{1} & D_{G+1} & \cdots & D_{(L-1) G+1} \\
\vdots & \vdots & \vdots & \vdots \\
D_{1}^{L-1} & D_{G+1}^{L-1} & \cdots & D_{(L-1) G+1}^{L-1}
\end{array}\right]} \\
\times\left[\begin{array}{cccc}
A_{1} & 0 & & \cdots \\
0 & A_{G+1} & & \cdots \\
0 & & \ddots & 0 \\
0 & \cdots & & A_{(L-1) G+1}
\end{array}\right]
\end{gathered}
$$

It's easy to prove that the product of the full rank block Vandermonde and full rank block diagonal matrix above has nonzero determinant and thus is of full rank $M L$. Since in $G(C, E)^{T}$ of $M L \times N_{c}$, we can find a submatrix of dimension $M L \times M L$ which is of full rank, we conclude that $\operatorname{rank}(S)=$ $\operatorname{rank}\left(G(C, E)^{T}\right)=M L$ is ensured, and the space-frequency codes proposed can achieve the maximum diversity gain of $M N L$.

\section{Space-Frequency Decoding}

Let $\mathbf{r}^{j}, \mathbf{w}^{j}$ denote the size $N_{c} \times 1$ received signal vector and noise vector at the $j$ th receive antenna, respectively. Now $\mathbf{r}^{j}$ can be divided into $L G$ size $M \times 1$ subvectors $\left\{\mathbf{r}^{j, k, g}\right\}$ for $g, k$ with the same definition as that in the section of code construction. Similarly, $\mathbf{w}^{j}$ can be divided into $L G$ size $M \times 1$ subvectors $\left\{\mathbf{w}^{j, k, g}\right\}$.

In addtion, $\theta_{i}^{T}$ denotes the $i$ th row of the CR matrix $\Theta$ and $H_{i, l}^{j}$ denotes the channel frequency response of the $i$ th transmit and $j$ th receive antenna pair at the $l$ th tone. Also, we define the diagonal matrix

$$
\Lambda_{k, g}^{j}=\operatorname{diag}\left\{\begin{array}{c}
H_{1,(k-1) G M+g M}^{j} \\
H_{2,(k-1) G M+g M+1}^{j} \\
\vdots \\
H_{M,(k-1) G M+(g+1) M-1}^{j}
\end{array}\right\}
$$

Therefore, for our SFBC, we can get

$$
\begin{aligned}
\mathbf{r}^{j} & =\left[\begin{array}{lllll}
\mathbf{r}^{j, 1,0} & \mathbf{r}^{j, 1,1} & \cdots & \mathbf{r}^{j, L, G-2} & \mathbf{r}^{j, L, G-1}
\end{array}\right]^{T} \\
& =\left[\begin{array}{lllll}
\mathbf{b}^{j, 1,0} & \mathbf{b}^{j, 1,1} & \cdots & \mathbf{b}^{j, L, G-2} & \mathbf{b}^{j, L, G-1}
\end{array}\right]^{T} \\
& +\left[\begin{array}{lllll}
\mathbf{w}^{j, 1,0} & \mathbf{w}^{j, 1,1} & \cdots & \mathbf{w}^{j, L, G-2} & \mathbf{w}^{j, L, G-1}
\end{array}\right]^{T}
\end{aligned}
$$

where

$$
\mathbf{b}^{j, k, g}=\Lambda_{k, g}^{j}\left[\begin{array}{l}
\theta_{(k-1) M+1}^{T} \\
\theta_{(k-1) M+2}^{T} \\
\vdots \\
\theta_{k M}^{T}
\end{array}\right] \mathbf{s}_{g}
$$

and $\mathbf{r}^{j, k, g}=\mathbf{b}^{j, k, g}+\mathbf{w}^{j, k, g}$.

Combining those sub-blocks in the $g$ th group, we get

$$
\begin{aligned}
& \mathbf{r}^{j, g}=\left[\begin{array}{llll}
\mathbf{r}^{j, 1, g} & \mathbf{r}^{j, 2, g} & \cdots & \mathbf{r}^{j, L, g}
\end{array}\right]^{T} \\
& =\underbrace{\left[\begin{array}{ccc}
\Lambda_{1, g}^{j} & & \\
& \ddots & \\
& & \Lambda_{L, g}^{j}
\end{array}\right]}_{\Lambda_{g}^{j}} \Theta \mathbf{s}_{g}+\mathbf{w}^{j, g}
\end{aligned}
$$

Furthermore, combining information from the $g$-th group over the $\mathrm{N}$ receive antennas using maximal ratio combining (MRC), we get

$$
\begin{aligned}
\mathbf{y}_{g} & =[\underbrace{\left(\sum_{j=1}^{N}\left(\Lambda_{g}^{j}\right)^{H} \Lambda_{g}^{j}\right)}_{\Sigma_{g}})^{-1 / 2} \\
& \times\left[\begin{array}{lll}
\left(\Lambda_{g}^{1}\right)^{H} & \cdots & \left(\Lambda_{g}^{N}\right)^{H}
\end{array}\right] \mathbf{r}_{g} \\
& =\Sigma_{g}^{1 / 2} \Theta \mathbf{s}_{g}+\eta_{g}
\end{aligned}
$$

Finally, ML detection using sphere decoding [10] [16] of order $2 \times L \times M$ is used to decode $\mathbf{s}_{g}$ from $\mathbf{y}_{g}$.

\section{Simulation Results AND CONCLUSiOnS}

In our simulations, an OFDM system conforming to the IEEE 802.16.3 standard was used, with FFT size of 256. Modulation symbols used were BPSK, 4QAM or 16QAM where the total average symbol energy on $\mathrm{M}$ transmit antennas $E_{s}=1$. The variance $\sigma^{2}$ of the white gaussian noise per dimension is adjusted by the formula $\sigma^{2}=\frac{1}{2} 10^{-\frac{S N R}{10}}$. The $L$-tap frequency selective channel for each transmit-receive antenna pair has independent and identical distributed complex Gaussian coefficients with total power $=1.10^{5}$ independent realizations are used to obtain the simulation results. A label such "SFBCSDM2N1L2uniQPSK" for a curve in Fig. 3 indicates that the frequency selective channel between each transmit-receive antenna pair has 2 i.i.d. taps with 1 receive and 2 transmit antennas, and that the proposed SF block code with QPSK modulation and sphere decoding was employed. In addition, we assume that $N_{c}=M \times G \times L$ where $N_{c}, L, M, G$ denote the number of tones, the maximum expected length of the channel impulse response (less than or equal to the cyclic prefix), transmit antennas and the number of groups the tones are divided into, respectively. For a given $M$, even when $L$ is large(hence small $G$ ), performance is unaffected since the code design ensures that the $L$ symbols from the same group transmitted from the same antenna experience uncorrelated fading. It is also clear to see that the diversity gain achieved increases with $L$. We then compare SFBCOFDM with CGDD-OFDM proposed in [17] and BLASTOFDM [15] when ML detection is used for all the three schemes. Also, we compare SFBC-OFDM with the coding scheme in 802.11a standard with 16QAM signalling and rate $1 / 2$ convolutional code using 1 transmit antenna and 


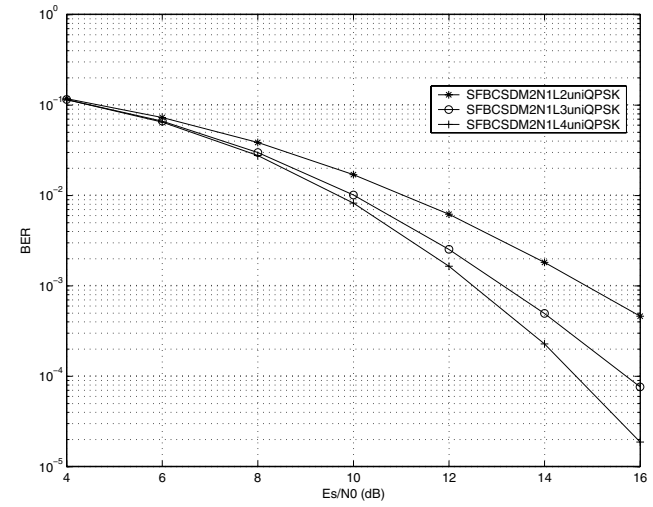

Fig. 3. BER performance of SFBC with different channel taps $(M=2, N=1)$

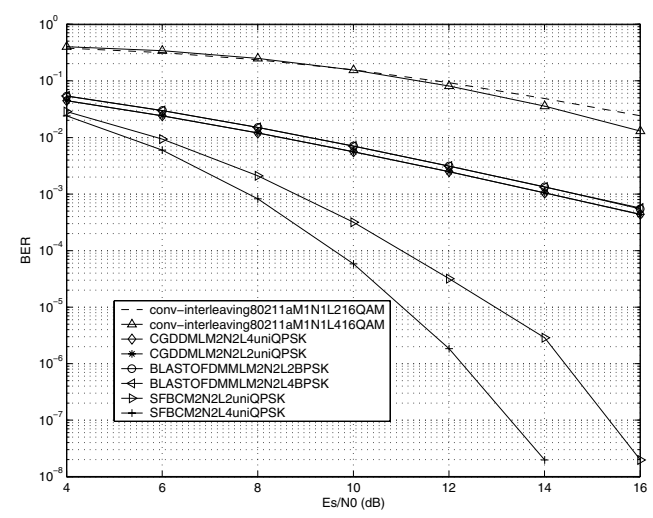

Fig. 4. BER performance comparision among CGDD-OFDM, BLASTOFDM, SFBC-OFDM with ML detection ( $M=2, N=2,64$ subcarriers)

1 receive antenna, to establish a performance baseline. At the receiver side, deinterleaving and Viterbi decoding are performed. In the simulations, the number of subcarriers was 64 and two transmit and receive antennas each were used for BLAST-OFDM,SFBC-OFDM and CGDD-OFDM. In order to preserve identical bit rates for a fair comparison, we chose BPSK for BLAST-OFDM, 16QAM for convolutional coding+interleaving, while using QPSK for CGDD-OFDM and SFBC-OFDM. From Fig.4, we can see that SFBC-OFDM outperforms the other three schemes as only it achieves the maximum diversity gain of $M N L$. For completeness, we also compared our method to the STFBC method proposed in [5], DPS proposed in [18] with two or four transmit antennas and one receive antenna. In order to keep the same bit rate, when four transmit antennas are used, we chose QPSK for STF, while using BPSK for both DPS and SFBC. From Fig.5, we find though STF can guarantee maximum coding gain, both DPS and SFBC have very similar performances; the gap between them is reduced as the number of transmit antennas increases. In addition, although the code matrices of DPS and SFBC are quite different, the performances of the two are almost the same. Since both of them need to use precoder of size $M L$, their decoding complexities are nearly identical as well.

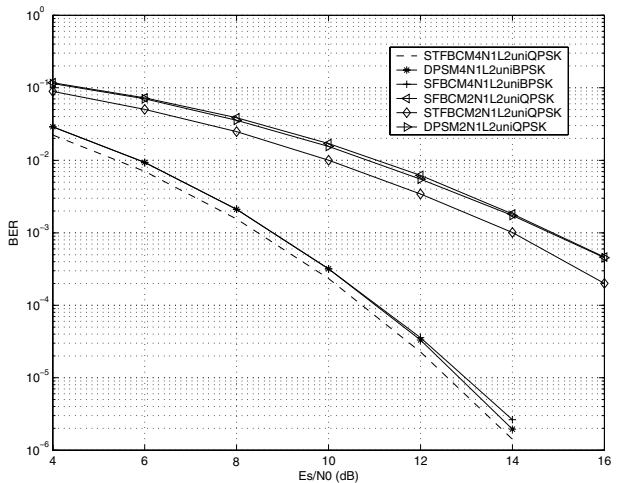

Fig. 5. BER performance comparision among STFBC [5] and SFBC-OFDM proposed and DPS[18]

\section{REFERENCES}

[1] Eklund, C.; Marks, R.B.; Stanwood, K.L.; Wang, S.,'IEEE standard 802.16: a technical overview of the WirelessMANTM air interface for broadband wireless access",IEEE Communications Magazine, Volume: 40 Issue: 6 , Jun 2002 Page(s): $98-107$

[2] IEEE 802.16-2001 "IEEE Standard for Local and Metropolitan Area Networks - Pt. 16: Air Interface for fixed Broadband Wireless Access Systems," Apr. 2002.

[3] H.Bolcskei, A.J.Paulraj, Space-frequency coded broadband OFDM systems, IEEE WCNC2000.

[4] H. Bolcskei, A.J. Paulraj, Space-frequency codes for broadband fading channels, IEEE ISIT2001.

[5] Z.Liu, Y. Xin, G.B. Giannakis, Space-time-frequency block coded OFDM with subcarrier grouping and constellation precoding, IEEE ICASSP2002.

[6] Y. Xin, Z. Wang, G.B. Giannakis,Space-time constellation-rotating codes maximizing diversity and coding gains, GLOBECOM2001.

[7] G.J. Foschini, et al., Simplified processing for wireless communication at high spectral efficiency, IEEE JSAC-WL. Commun. Series, vol.17, no.11, Nov. 1999, pp. 1841-52.

[8] L. Zheng, D. N.C. Tse, Optimal diversity-multiplexing tradeoff in multiple-antenna channels. In Proceeding of the 39th Allerton Conference on Communication, Control and Computing, Monticello, IL, Oct.2001;pp835-844.

[9] D. Agrawal, V. Tarokh, A. Naguib, N. Seshadri, Space-time coded OFDM for high data-rate wireless communication over wideband channels, Proc. of VTC, Ottawa, Ont., Canada., 18-21 May, 1998. pp2232-2236.

[10] M.O. Damen, A. Chkeif, and J.-C. Belfiore, Lattice codes decoder for space-time codes, IEEE Communications Letters, vol.4, no.5,pp161-163, May 2000.

[11] King F. Lee and Douglas B. Williams, A space-frequency transmitter diversity technique for OFDM systems, GLOBECOM'2000.

[12] Z. Liu, G.B. Giannakis, S.Zhou, B. Muquet, Space-time coding for broadband wireless communications, Wirel.Commun. Mob.Comput. 2001:1:35-53

[13] P.Joo, J. Son, D.Kang, Analysis of STFBC-OFDM for BWA in SUI channel, IEEE C802.16a-02/18, Samsung Electronics,2002-01-22.

[14] S.M. Alamouti, A simple transmit diversity technique for wireless communications, IEEE JSAC 1998, 16(8): 1451-1458.

[15] R.J. Piechocki, P.N. Fletcher, A.R. Nix, C.N. Canagarajah, and J.P. McGeehan, Performance evaluation of BLAST-OFDM enhanced Hiperlan/2 using simulated and measured channel data. Electronics Letters, 30th Aug. 2001, vol.37, No.18.

[16] A.M.Chan, I. Lee, A new reduced-complexity sphere decoder for multiple antenna systems, ICC02.

[17] D.Gore, S. Sandhu, A. Paulraj,Delay diversity codes for frequency selective channels, ICC02.

[18] Xiaoli Ma and G.B. Giannakis, Space-time-multipath coding using digital phase sweeping, Globecom2002. 